\title{
Diabetes mellitus e cancro colorretal: uma revisão baseada na evidência
}

Sandra Esteves Oliveira,* Isabel Machado,* Alfredo João Pereira,* Marlene Barros*

\section{RESUMO}

Objetivo: A diabetes mellitus (DM) e o cancro colorretal (CCR) são patologias com incidência crescente e elevada morbimortalidade. Segundo dados da International Diabetes Federation, a DM foi responsável, em 2014, por 4,9 milhões de mortes a nível mundial. Alguns estudos sugerem a DM como fator de risco para CCR. Contudo, esta associação permanece inconsistente. Assim, o objetivo deste estudo foi rever a evidência atualmente disponível sobre a associação entre DM e aumento da incidência e mortalidade por CCR. Fontes de dados: National Guideline Clearing House, NICE, Canadian Medical Association Practice Guidelines, Cochrane, PubMed, Direção-Geral da Saúde.

Métodos de revisão: Pesquisa de meta-análises (MA), revisões sistemáticas (RS), estudos observacionais e normas de orientação clínica/guidelines baseadas na evidência, publicadas nos últimos cinco anos, em português, espanhol e inglês, utilizando os termos MeSH diabetes mellitus e cancer, colorectal e os correspondentes DeCS. Foi utilizada a escala SORT (Strength of Recommendation Taxonomy), da American Academy of Family Physicians, para atribuição dos níveis de evidência e forças de recomendação.

Resultados: Obtiveram-se 48 artigos, sendo que seis MA e um estudo observacional cumpriram os critérios de inclusão. Os estudos incluídos demonstraram resultados estatisticamente significativos, consistentes com o aumento da incidência e mortalidade por CCR em indivíduos diabéticos. Quatro MA realizaram uma análise independente por local da neoplasia (cólon e reto) e verificaram um aumento estatisticamente significativo da incidência e mortalidade dos dois tipos de cancro na população diabética. Da mesma forma, quatro MA realizaram uma análise de subgrupo por género, verificando-se, na maioria dos estudos, uma associação positiva entre DM e aumento da incidência de CCR em ambos os géneros.

Conclusão: A evidência atualmente disponível demonstra um aumento estatisticamente significativo da incidência e mortalidade por CCR em indivíduos diabéticos (SORT A). No entanto, são necessários estudos mais homogéneos em relação à metodologia, que considerem o subtipo de DM, as diferenças consoante a etnia, a interferência de fatores confundidores como a dieta, obesidade, tabagismo, sedentarismo, medicação antidiabética e a duração da doença. Estes resultados são clinicamente relevantes e podem ter implicações no âmbito de saúde pública, dada a elevada prevalência e morbimortalidade da DM e CCR, bem como a possível base fisiopatológica comum entre estas duas entidades.

Palavras-chave: Diabetes mellitus; Cancro colorretal.

\section{INTRODUÇÃO}

A diabetes mellitus (DM) e o cancro colorretal (CCR) são patologias frequentes, sobretudo nos países desenvolvidos. A incidência de DM tem vindo a aumentar, a par do aumento da incidência da obesidade, assumindo-se como um grave problema de saúde pública. Segundo dados da International Diabetes Federation, a prevalência de DM na Europa é de $7,9 \%$, afetando cerca de 387 milhões de pessoas. ${ }^{1}$ Estima-se um aumento de 205 milhões de novos casos em 2035.

* Médicos Internos de Medicina Geral e Familiar

Unidade de Saúde Familiar do Minho, ACES Cávado I - Braga
Constitui uma das principais causas de morbilidade e mortalidade, tendo sido responsável, em 2014, por 4,9 milhões de mortes a nível mundial. ${ }^{1}$ Em Portugal, segundo dados do Instituto Nacional de Estatística, a DM esteve na origem de $4,3 \%$ das mortes ocorridas em 2013, o que corresponde a uma perda de 4.683 anos potenciais de vida. ${ }^{2}$

O CCR é o $3 .^{\circ}$ cancro mais frequente no homem e o $2 .^{\circ}$ na mulher e é a $3 .^{a}$ causa mais frequente de morte, após o cancro da mama e do pulmão. ${ }^{3}$ Pensa-se que a DM tipo 2 possa estar associada a CCR devido ao facto de partilharem vários fatores de risco, como estilos de vida (sedentarismo, tabagismo, dieta pobre em fruta, vegetais ou fibras e rica em carnes vermelhas, gorduras saturadas) e obesi- 
dade. ${ }^{4-7}$ Alguns estudos sugerem, como mecanismo subjacente à carcinogénese, a hiperinsulinemia que ocorre na DM tipo 2 devido à insulinorresistência. ${ }^{5}$

Em 2005 surgiu a primeira meta-análise (MA), de 15 estudos (seis estudos caso-controlo e nove estudos coorte), que demonstrou a associação entre DM tipo 2 e risco aumentado de CCR. ${ }^{8}$ Nos últimos anos, novos estudos coorte debruçaram-se sobre esta associação. No entanto, alguns estudos demonstraram resultados inconsistentes relativamente à força desta associação ${ }^{9-13}$ e permanece a incerteza em relação ao impacto da DM na mortalidade específica por CCR, bem como na incidência reportada por sexos. ${ }^{13-15}$

As guidelines americanas e europeias alertam para a associação do tabagismo e obesidade com CCR, mas não realçam o risco em doentes com DM..$^{16-17}$

Deste modo, este estudo teve como objetivo rever a evidência mais atual sobre a associação entre DM e aumento da incidência e mortalidade por CCR.

\section{MÉTODOS}

Foi realizada uma pesquisa bibliográfica em maio de 2015, por dois autores da revisão, utilizando os termos MeSH diabetes mellitus e cancer, colorectal com $100 \%$ de concordância. Foi selecionada a pesquisa de MA, revisões sistemáticas (RS), estudos observacionais e normas de orientação clínica/guidelines baseadas na evidência nas bases de dados da National Guideline Clearing House, NICE, Canadian Medical Association Practice Guidelines, Direção-Geral da Saúde, Cochrane, PubMed, dos artigos publicados entre janeiro de 2010 e maio de 2015, nas línguas portuguesa, espanhola e inglesa. A população em estudo incluiu indivíduos com idade igual ou superior a 18 anos. As variáveis em estudo foram os diagnósticos de DM e CCR, a sobrevida e a mortalidade. Os outcomes avaliados foram, respetivamente, o aumento da incidência e mortalidade por CCR nos doentes com diagnóstico de DM. Foram incluídos estudos que avaliaram mortalidade por todas as causas, mortalidade específica por CCR, sobrevida sem doença ou recorrência da doença e ainda estudos que avaliaram a incidência/mortalidade de CCR reportada consoante $o$ sexo e local da neoplasia (cólon e reto). Foram excluídos os indivíduos com idade inferior a 18 anos, antecedentes pessoais de doenças inflamatórias intestinais ou CCR e os estudos que não comparavam com os grupos controlo ou que tinham outros outcomes, como aumento da incidência de pólipos benignos ou qualidade de vida. Utilizou-se a escala SORT (Strength of Recommendation Taxonomy), da American Academy of Family Physicians, para atribuição dos níveis de evidência (NE) e forças de recomendação.

\section{RESULTADOS}

Foram obtidos 48 artigos, tendo sido selecionadas seis MA e um estudo observacional. Vinte e oito artigos foram excluídos com base no título, um após leitura do resumo e seis por repetição. Após leitura completa, três foram excluídos por não cumprirem os critérios de inclusão e três por se reportarem a MA com estudos referenciados noutras MA já incluídas (Figura 1).

A descrição resumida dos artigos incluídos encontra-se no Quadro 1.

A MA de Jiang Y, et a ${ }^{5}$ (NE 1), publicada em 2011, teve como principal objetivo avaliar a relação entre a DM e a incidência e mortalidade por CCR. Esta MA incluiu 41 estudos coorte, com um tempo médio de follow-up de cerca de 10,5 anos, totalizando cerca de 3 milhões de indivíduos diabéticos com idades compreendidas entre os 25 e os 84 anos. Verificaram que existia uma associação positiva entre DM e incidência de CCR (RR-risco relativo de 1,27), bem como entre DM e mortalidade por CCR (RR 1,20). Restringindo a MA a estudos controlados para fatores confundidores (índice de massa corporal, tabaco e atividade física), verifica-

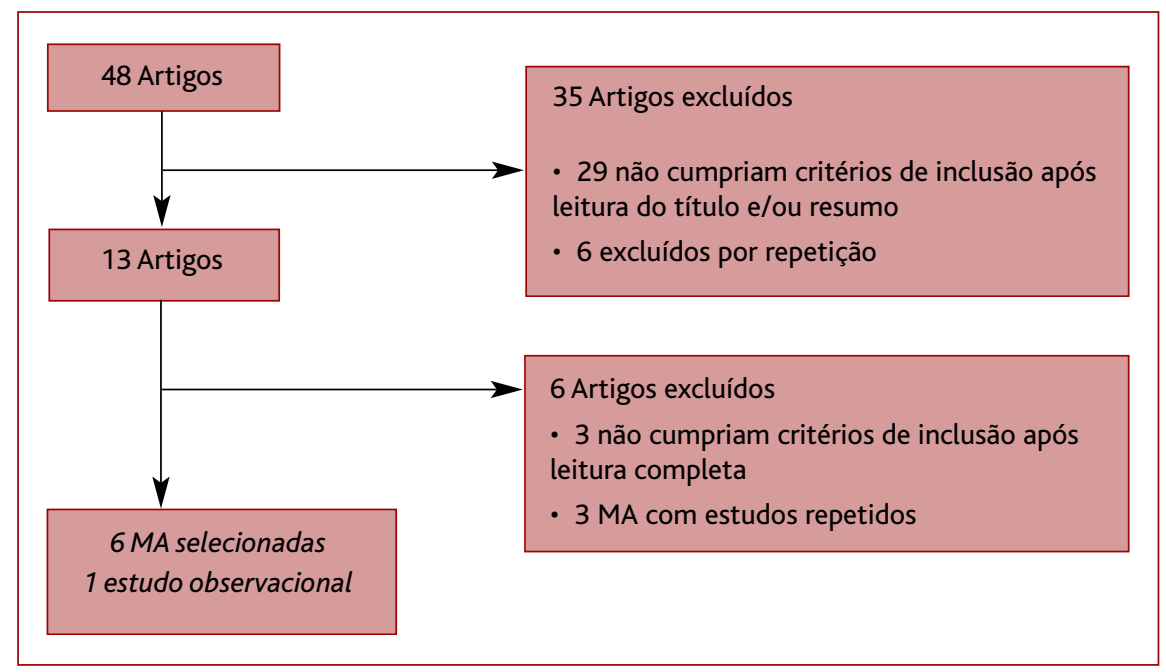

Figura 1. Esquema da seleção dos estudos. 


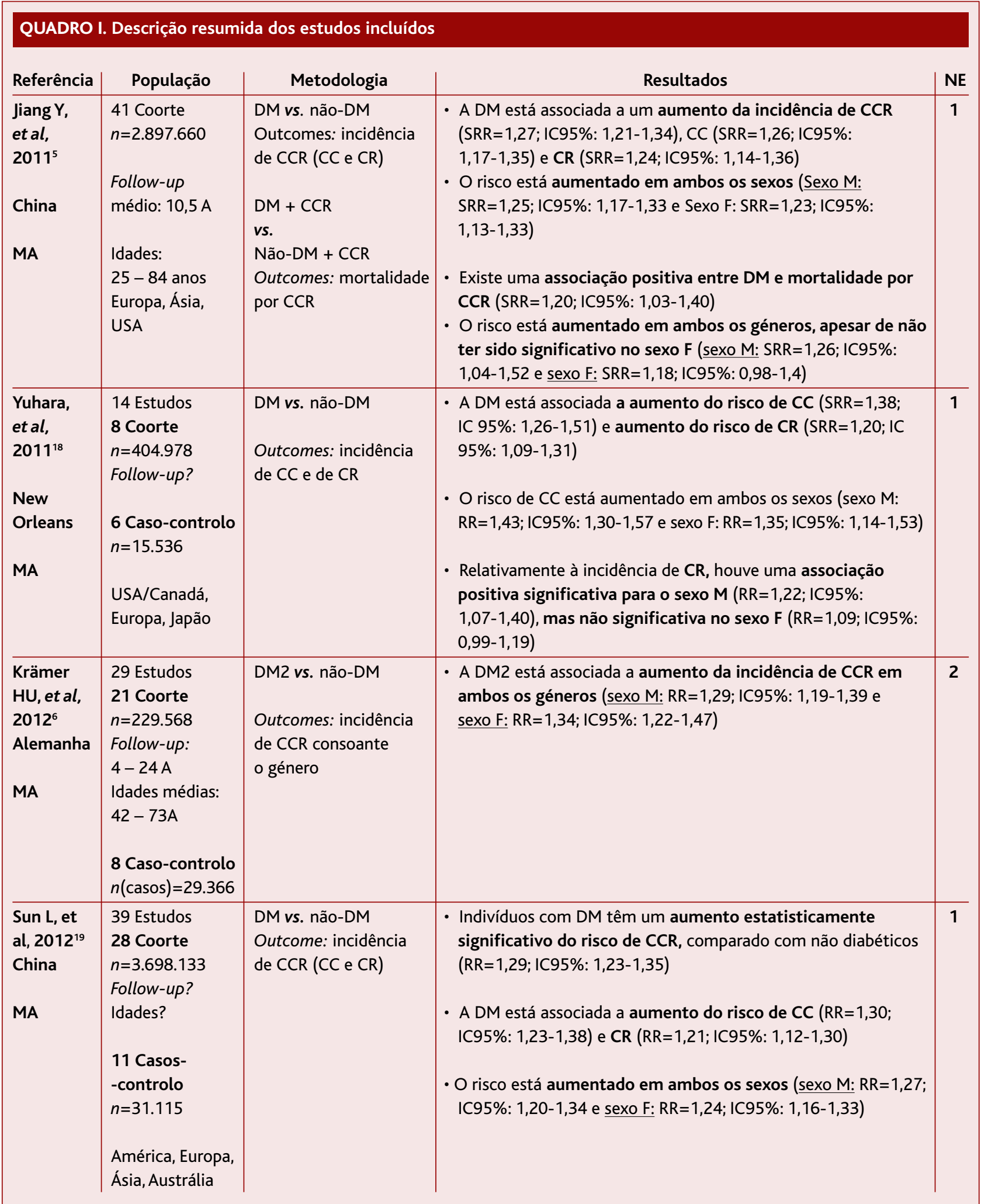




\begin{tabular}{|c|c|c|c|c|}
\hline Referência & População & Metodologia & Resultados & NE \\
\hline $\begin{array}{l}\text { Mills KT, } \\
\text { et al, } \\
2013^{14} \\
\text { New } \\
\text { Orleans } \\
\text { MA }\end{array}$ & $\begin{array}{l}26 \text { Coorte } \\
n=214.303 \\
\text { Follow-up médio: } \\
2 \mathrm{~A}-9 \mathrm{~A} \\
\text { Idades médias: } \\
50,8-77,2 \\
\text { USA, Europa, } \\
\text { Ásia, Nova } \\
\text { Zelândia }\end{array}$ & $\begin{array}{l}\text { DM + CCR } \\
\text { vs. } \\
\text { não-DM + CCR } \\
\text { Outcomes: mortalidade } \\
\text { por todas as causas, } \\
\text { mortalidade específica } \\
\text { por CCR e sobrevida } \\
\text { livre de doença }\end{array}$ & $\begin{array}{l}\text { - Doentes com CCR e DM têm maior mortalidade por todas as } \\
\text { causas ( } R R=1,17 ; \text { IC95\%: } 1,09-1,25) \text { e maior mortalidade } \\
\text { específica por CCR ( } R R=1,12 ; \text { IC95\%: } 1,01-1,24) \text { e menor } \\
\text { sobrevida sem doença ( } R R=1,54 ; \text { IC95\%: } 1,08-2,18) \\
\text { - DM está associada a maior mortalidade específica por CC } \\
\text { (RR=1,22, IC95\%: 1,08-1,39) e por CR (RR=1,23, IC95\%: } \\
1,13-1,33)\end{array}$ & 1 \\
\hline $\begin{array}{l}\text { Bruijn KM, } \\
\text { et al, } \\
2013^{15} \\
\text { Holanda } \\
\text { MA }\end{array}$ & $\begin{array}{l}20 \text { Estudos } \\
1 \text { ECAC; } 2 \text { RS } \\
17 \text { Coorte } \\
n=1.930 .309 \\
\text { Follow-up médio: } \\
4-26 \text { A } \\
\text { Idades? } \\
\text { USA, UK, } \\
\text { Holanda, Suécia, } \\
\text { Finlândia, Escócia }\end{array}$ & $\begin{array}{l}\text { DM vs. não-DM } \\
\text { Outcome: incidência } \\
\text { de CCR } \\
\text { DM + CCR vs. } \\
\text { não-DM + CCR } \\
\text { Outcome: mortalidade } \\
\text { específica por CCR }\end{array}$ & $\begin{array}{l}\text { - A DM está associada a maior incidência de CCR (HR=1,26, } \\
1,14-1,40 ; p<0,001) \\
\text { - A DM está associada a risco acrescido de mortalidade } \\
\text { específica por } C C R(H R=1,30,1,15-1,47 ; p<0,001)\end{array}$ & 1 \\
\hline $\begin{array}{l}\text { Peeters PJ, } \\
\text { et al, } \\
2015^{20} \\
\text { UK } \\
\text { Estudo } \\
\text { observa- } \\
\text { cional }\end{array}$ & $\begin{array}{l}\text { Estudo coorte } \\
\text { Retrospetivo } \\
n=300.039 \mathrm{DM} 2 \\
\text { Idade média: } 61 \mathrm{~A} \\
\text { Follow-up médio: } \\
4,5 \mathrm{~A}\end{array}$ & $\begin{array}{l}\text { DM vs. não-DM } \\
\text { Outcome: incidência } \\
\text { de CCR }\end{array}$ & $\begin{array}{l}\text { - Aumento moderado do risco de CCR nos diabéticos tipo } 2) \\
\text { (HR=1,26; IC95\%: } 1,18-1,33) \\
\text { - Não houve uma tendência clara para aumento do risco de CCR } \\
\text { com a progressão do estadio de tratamento da DM }\end{array}$ & 2 \\
\hline
\end{tabular}

Legenda: $C C=$ cancro cólon; $C C R=$ cancro colorretal; $C R=$ cancro reto; $D M=$ diabetes mellitus; $E C A C=$ ensaios clínicos controlados e aleatorizados; $F=$ feminino; $\mathrm{HR}=$ hazard ratio; $\mathrm{IC}=$ intervalo confiança; $M=$ masculino; $M A=$ eta-análises; $N E=$ nível de evidência; $N E$ 1=estudo de boa qualidade, evidência orientada para o doente; NE 2=estudo de qualidade limitada, evidência orientada para o doente; RR=risco relativo; RS=revisões sistemáticas; $S R R=$ risco relativo sumário.

ram que se manteve uma associação positiva. Verificaram ainda um aumento da incidência de CCR em indivíduos diabéticos de ambos os géneros e de forma similar. No entanto, em relação à mortalidade por CCR, não verificaram resultados estatisticamente significativos no sexo feminino.

No mesmo ano foi publicada a MA de Yuhara, et al ${ }^{18}$ (NE 1) que teve como principal objetivo avaliar a relação entre
DM e incidência de CC e CR, separadamente. Trata-se de uma MA de menor dimensão, com oito estudos coorte e seis estudos caso-controlo. Uma vez que o CCR é considerado, em termos absolutos, uma doença rara, os resultados de ambos os estudos podem ser comparáveis. ${ }^{18}$ Os autores verificaram uma associação positiva, estatisticamente significativa entre DM e aumento da incidência 
para os dois tipos de cancro. O risco demonstrou-se aumentado em ambos os sexos para CC. No entanto, os resultados não foram estatisticamente significativos para o risco de CR no sexo feminino. Realizando uma análise independente aos estudos de melhor qualidade incluídos nesta MA, os autores não verificaram alteração da magnitude dos resultados. Da mesma forma que na MA anterior, realizando uma análise restrita a estudos controlados para tabagismo e obesidade, a associação entre DM e CCR manteve-se positiva, o que, segundo os autores, indica que a DM possa ser um fator de risco independente para CCR.

Em 2012 foi publicada a MA de Krämer, HU et al ${ }^{6}$ (NE 2) que teve como principal objetivo estudar a relação entre DM tipo 2 e incidência de CCR consoante o género. Incluiu 21 estudos coorte e oito estudos caso-controlo. Os primeiros com um tempo de follow-up que variou entre os quatro e os 24 anos, com indivíduos com idades compreendidas entre os 42 e 73 anos. Os autores verificaram que a DM tipo 2 está associada a aumento de incidência de CCR em ambos os géneros de forma similar. No entanto, os estudos incluídos nesta MA eram bastante heterogéneos e os riscos diferiram ligeiramente quando foi efetuada uma análise independente dos estudos coorte $v s$. caso-controlo, tendo sido superior nos últimos.

A MA de Sun \&Yu ${ }^{19}$ (NE 1), publicada em 2012, teve como um dos principais objetivos avaliar a associação entre DM e o risco de CCR e investigar se essa associação era dependente do sexo. Incluíram 28 estudos coorte com cerca de quatro milhões de indivíduos diabéticos e 11 estudos de caso-controlo. Verificaram que, em comparação com a população não diabética, o risco relativo de CCR nos diabéticos era de 1,29. A análise independente por subgrupos revelou um risco significativamente aumentado de CCR para ambos os sexos.

Mais recentemente, em 2013, foi publicada a MA de Mills $K T$, et $a l^{14}$ (NE 1), que avaliou a associação entre indivíduos com DM e a mortalidade específica por CCR, mortalidade por todas as causas e sobrevida livre de doença. Os autores analisaram 26 estudos coorte, perfazendo um total de 214.303 indivíduos. Verificaram que os indivíduos diabéticos com CCR têm um risco significativamente aumentado de mortalidade por todas as causas (RR 1,17), maior mortalidade específica por CCR (RR 1,12) e menor sobrevida livre de doença (RR 1,54). Uma vez que o prognóstico de CCR difere consoante a presença de metástases, foi realizada uma análise de sensibilidade restrita aos cancros não metastáticos e foi observada uma associação também positiva entre DM e mortalidade por CCR nestes casos (RR 1,25, 95\% IC:1,06, 1,52).

A MA de Bruijn KMJ et al ${ }^{15}$ (NE 1), publicada também em
2013, teve como principais objetivos avaliar a associação entre indivíduos com DM e a incidência e mortalidade por CCR. Os autores examinaram 20 estudos, na sua maioria estudos de coorte prospetivos, com cerca de dois milhões de indivíduos com um tempo médio de follow-up que variou entre os quatro e os 26 anos. Não houve heterogeneidade entre os estudos que reportaram a incidência por CCR, contrariamente aos estudos que reportaram a mortalidade por CCR. Os autores verificaram uma associação positiva, estatisticamente significativa entre DM e aumento da incidência e mortalidade por CCR.

A par destas MA, foi incluído um estudo de coorte retrospetivo (NE2), publicado em $2015,{ }^{20}$ que teve como principais objetivos avaliar o risco de CCR em diabéticos tipo 2, bem como verificar se existe uma associação com o estadio de tratamento para a DM. Para tal definiram diferentes estadios de tratamento, desde o tratamento com apenas um antidiabético ao tratamento com insulina em monoterapia. Os autores estudaram uma população de 300.039 diabéticos tipo 2, com um tempo médio de follow-up de 4,5 anos. Verificaram um aumento moderado do risco de CCR nos diabéticos tipo 2 (HR 1,26). No entanto, não houve uma associação estatisticamente significativa entre a progressão do tratamento da DM e o aumento de risco de CCR.

\section{CONCLUSÃO}

A evidência atualmente disponível é consistente relativamente ao aumento moderado da incidência e mortalidade por CCR em indivíduos diabéticos (SORT A).

Para explicar esta associação põe-se a hipótese de que a hiperinsulinemia possa ser prejudicial por duas vias. Uma, através da ativação direta da insulina e do fator de crescimento insulin-like tipo 1 (IGF-1), que promovem a proliferação de células do cólon, modulando o seu metabolismo e inibindo a apoptose de células normais ou cancerígenas. Outra via, através da inibição das proteínas de ligação ao IGF, aumentando, assim, a biodisponibilidade do IGF-1. ${ }^{21}$ Além disso, a insulinorresistência conduz ao aumento de mediadores pró-inflamatórios (TNF- $\alpha$, IL-6 e leptina) que favorecem a inflamação e, consequentemente, aumentam o risco de CCR. A par deste mecanismo, a obstipação/trânsito intestinal lento, que ocorre nos diabéticos, pode conduzir ao aumento do tempo de exposição a substâncias tóxicas potencialmente carcinogénicas (ácidos biliares fecais) que podem provocar alterações na cadeia de DNA. ${ }^{5}$

Relativamente à diferença por género, pensa-se que o risco poderá ser inferior no sexo feminino devido à interação entre a insulina e os estrogénios, uma vez que os es- 
trogénios podem minorar os efeitos deletérios da hiperinsulinemia e diminuir o risco de CCR. ${ }^{22}$ No entanto, na presente revisão, das quatro MA que efetuaram uma análise independente por géneros, apenas numa MA o risco de incidência de CCR/CC/CR não estava significativamente aumentado no sexo feminino, ${ }^{18}$ tendo sido similar em ambos os géneros em três das MA analisadas. Serão necessários mais estudos para clarificar esta questão. De salientar também que a medida mais precisa para se avaliar o tempo até ter o evento é o Hazard Ratio, o que só foi possível obter em dois dos estudos incluídos.

As diferenças encontradas podem ser explicadas pelas limitações que são transversais, na generalidade, em todas as MA. A primeira prende-se com o viés de publicação inerente às MA, uma vez que os estudos de pequena dimensão ou com resultados pouco significativos tendem a não ser publicados. Por motivos éticos, a questão clínica não poderia ser respondida através de estudos experimentais, pelo que todos os estudos incluídos nas MA constituem estudos observacionais, com menor força relativamente aos primeiros. O viés de diagnóstico de DM é também relevante, dado que na maioria dos estudos incluídos a DM foi maioritariamente autorreportada e não apenas baseada em registos médicos, logo, pode ter sido subdiagnosticada e indivíduos diabéticos podem ter sido alocados ao grupo controlo. A heterogeneidade das populações estudadas em relação à etnia, subtipo de diabetes (na maioria dos estudos, não referido), heterogeneidade relativamente ao controlo de fatores confundidores, como a dieta, obesidade, tabagismo, sedentarismo, são outras limitações apontadas. Além disso, nem todas as MA realizaram ajuste das populações para a idade, o que seria uma mais-valia, bem como o ajuste das populações para a medicação antidiabética, uma vez que existe evidência de que a metformina tem um efeito protetor na mortalidade por CCR e de que a insulinoterapia pode aumentar esse risco. ${ }^{15}$ Além destes vieses, o aumento da incidência de CCR pode estar relacionado com o facto dos indivíduos diabéticos serem mais vigiados e poderem ter acesso a mais oportunidades de rastreio. Da mesma forma, o aumento da mortalidade por CCR pode estar relacionado com uma possível menor resposta à quimioradioterapia nos indivíduos diabéticos. ${ }^{18}$ Por último, uma vez que a DM por si só aumenta o risco cardiovascular, as conclusões relativas ao efeito da DM na mortalidade específica por cancro são limitadas. ${ }^{14}$ Desta forma, de modo a determinar o papel da DM no prognóstico do CCR, deve ser também estudada a mortalidade específica do cancro, sobrevida livre de doença e a recorrência, o que não se verificou na maioria dos estudos.
Adicionalmente são necessários estudos que avaliem a associação entre a duração da DM e o risco de CCR, bem como o tempo até terem o evento, uma vez que, com a evolução da DM e a diminuição da produção de insulina endógena, o risco de CCR poderá eventualmente ser inferior. Dos estudos incluídos, apenas no estudo observacional foi feita uma tentativa de estadiar o tratamento da DM e, possivelmente, medir a insulinorresistência ao longo da duração da doença, não se tendo verificado relação entre a progressão do tratamento e o aumento do risco de CCR. No entanto, são necessários mais estudos que explorem esta questão.

Não obstante, estes resultados têm importância clínica e podem ter implicações em saúde pública à escala global através da implementação de políticas de saúde com intervenção, por exemplo, ao nível dos estilos de vida, devido à possível base fisiopatológica comum entre a DM e o CCR e ao facto de serem entidades muito frequentes e com elevada morbimortalidade.

Assim, a DM pode constituir um fator de risco para CCR. Alguns autores defendem a revisão das guidelines de referência de modo a incluírem a DM como fator de risco e a determinar o período mais apropriado para o rastreio de CCR. ${ }^{18}$

Futuramente serão necessários estudos mais uniformes em relação à metodologia, nomeadamente com recurso a registos médicos para estratificar os grupos controlo vs. intervenção, subtipo de DM e que permitam ajustar possíveis fatores confundidores (obesidade, tabagismo, sedentarismo, dieta hiperlipídica).

\section{REFERÊNCIAS BIBLIOGRÁFICAS}

1. International Diabetes Federation. Diabetes atlas. 6th ed. Brussels: IDF; 2015. Available from: http://www.idf.org/diabetesatlas/

2. Instituto Nacional de Estatística. Diabetes mellitus. Lisboa: INE; 2014. Available from: https://www.ine.pt

3. Ferlay J, Soerjomataram I, Ervik M, Dikshit R, Eser S, Mathers C, et al. GLOBOCAN 2012 v1.0: cancer incidence and mortality worldwide [Internet]. Lyon: International Agency for Research on Cancer; 2013. Available from: http://globocan.iarc.fr

4. Wu L, Yu C, Jiang H, Tang J, Huang HL, Gao J, et al. Diabetes mellitus and the occurrence of colorectal cancer: an updated meta-analysis of cohort studies. Diabetes Technol Ther. 2013;15(5):419-27.

5. Jiang Y, Ben Q, Shen H, Lu W, Zhang Y, Zhu J. Diabetes mellitus and incidence and mortality of colorectal cancer: a systematic review and metaanalysis of cohort studies. Eur J Epidemiol. 2011;26(11):863-76.

6. Krämer HU, Schöttker B, Raum E, Brenner H. Type 2 diabetes mellitus and colorectal cancer: meta-analysis on sex-specific differences. Eur J Cancer. 2012;48(9):1269-82

7. Deng L, Gui Z, Zhao L, Wang J, Shen L. Diabetes mellitus and the incidence of colorectal cancer: an updated systematic review and meta-analysis. Dig Dis Sci. 2012;57(6):1576-85.

8. Larsson SC, Orsini N, Wolk A. Diabetes mellitus and risk of colorectal cancer: a meta-analysis. J Natl Cancer Inst. 2005;97(22):1679-87.

9. Hu FB, Manson JE, Liu S, Hunter D, Colditz GA, Michels KB, et al. Prospecti- 
ve study of adult onset diabetes mellitus (type 2) and risk of colorectal cancer in women. J Natl Cancer Inst. 1999;91(6):542-7.

10. Larsson SC, Giovannucci E, Wolk A. Diabetes and colorectal cancer incidence in the cohort of Swedish men. Diabetes Care. 2005;28(7):1805-7.

11. Bowers K, Albanes D, Limburg P, Pietinen P, Taylor PR, Virtamo J, et al. A prospective study of anthropometric and clinical measurements associated with insulin resistance syndrome and colorectal cancer in male smokers. Am J Epidemiol. 2006;164(7):652-64.

12. Schoen RE, Tangen CM, Kuller LH, Burke GL, Cushman M, Tracy RP, et al. Increased blood glucose and insulin, body size, and incident colorectal cancer. J Natl Cancer Inst. 1999;91(13):1147-54.

13. Luo W, Cao Y, Liao C, Gao F. Diabetes mellitus and the incidence and mortality of colorectal cancer: a meta-analysis of twenty four cohort studies. Colorectal Dis. 2011 Nov 5. doi: 10.1111/j.1463-1318.2011.02875.x. [Epub ahead of print]

14. Mills KT, Bellows CF, Hoffman AE, Kelly TN, Gagliardi G. Diabetes mellitus and colorectal cancer prognosis: a meta-analysis. Dis Colon Rectum. 2013;56(11):1304-19.

15. De Bruijn KM, Arends LR, Hansen BE, Leeflang S, Ruiter R, van Eijck CH. Systematic review and meta-analysis of the association between diabetes mellitus and incidence and mortality in breast and colorectal cancer. Br J Surg. 2013;100(11):1421-9.

16. Dominic OG, McGarrity T, Dignan M, Lengerich EJ.American College of Gastroenterology guidelines for colorectal cancer screening 2008. Am J Gastroenterol. 2009;104(10):2626-7.

17. Quirke P, Risio M, Lambert R, von Karsa L, Vieth M, International Agency for
Research on Cancer. European guidelines for quality assurance in colorectal cancer screening and diagnosis. First Edition - Quality assurance in pathology in colorectal cancer screening and diagnosis. Endoscopy. 2012;44 Suppl 3:SE116-30.

18. Yuhara H, Steinmaus C, Cohen SE, Corley DA, Tei Y, Buffler PA. Is diabetes mellitus an independent risk factor for colon cancer and rectal cancer? Am J Gastroenterol. 2011;106(11):1911-21.

19. Sun $L$, Yu S. Diabetes mellitus is an independent risk factor for colorectal cancer. Dig Dis Sci. 2012;57(6):1586-97.

20. Peeters PJ, Bazelier MT, Leufkens HG, de Vries F, De Bruin ML. The risk of colorectal cancer in patients with type 2 diabetes: associations with treatment stage and obesity. Diabetes Care. 2015;38(3):495-502.

21. Smith CJ, McKay GA, Fisher M. Diabetes, colorectal cancer and cyclooxygenase 2 inhibition. Int J Clin Pract. 2008;62(5):810-5.

22. Giovannucci E. Insulin, insulin-like growth factors and colon cancer: a review of the evidence. J Nutr. 2001;131(11 Suppl):3109S-20S.

\section{CONFLITO DE INTERESSES}

Os autores declaram não ter conflitos de interesses.

\section{ENDEREÇO PARA CORRESPONDÊNCIA}

Sandra Esteves Oliveira

E-mail: sandrafeoliveira@gmail.com

Recebido em 05-09-2015

Aceite para publicação em 14-02-2016

\section{ABSTRACT}

\section{DIABETES MELLITUS AND COLORECTAL CANCER: AN EVIDENCE-BASED REVIEW}

Objective: Diabetes mellitus (DM) and colorectal cancer (CRC) are diseases with increasing incidence and high mortality. In 2014, DM accounted for 4.9 million deaths worldwide according to the International Diabetes Federation. Some studies suggest that DM is a risk factor for CRC. However, this association is inconsistent. The aim of this study was to review the evidence for the association between DM and increased incidence and mortality from CRC.

Data sources: National Guideline Clearing House, NICE, Canadian Medical Association Practice Guidelines, Cochrane, PubMed, and Direção-Geral da Saúde.

Methods: We searched for meta-analyses (MA), systematic reviews (SR), observational studies, and clinical guidelines based on evidence published in the last five years in Portuguese, Spanish and English, using the MeSH terms 'diabetes mellitus' and 'cancer, colorectal' and the corresponding DeCS (Portuguese search terms). We used the Strength of Recommendation Taxonomy (SORT) scale of the American Academy of Family Physicians to assign levels of evidence and strength of recommendations.

Results: From the 48 articles obtained, $6 \mathrm{MA}$ and 1 observational study met the inclusion criteria. Included studies found statistically significant results showing increased mortality and incidence of CRC in diabetic individuals. Four MA conducted an independent analysis by site of cancer (colon and rectum) and found a statistically significant increase in incidence and mortality of both types of cancer in diabetic population. Similarly, four MA conducted a subgroup analysis by gender showing, in most studies, a positive association between diabetes and increased incidence of CRC in both genders.

Conclusion: The currently available evidence shows a statistically significant and moderate increase in incidence and mortality from CRC in diabetic individuals (SORT A). We need studies with more homogeneous methodology, regarding subtypes of DM, ethnicity, and the effects of confounding factors such as diet, obesity, smoking, sedentary lifestyle, antidiabetic medication and duration of the disease. These results are clinically relevant and may have public health implications, given the high prevalence and morbidity of DM and CRC and the possible common pathophysiological basis of these diseases.

Keywords: Diabetes mellitus; Colorectal cancer. 